University of Nebraska - Lincoln

DigitalCommons@University of Nebraska - Lincoln

1988

\title{
Diet Choices of Blue Jays (Cyanocitta cristata) as a Function of Time Spent Foraging
}

Sonja I. Yoerg

University of Massachusetts, Amherst

Alan C. Kamil

University of Massachusetts, Amherst

Follow this and additional works at: https://digitalcommons.unl.edu/bioscibehavior

Part of the Behavior and Ethology Commons

Yoerg, Sonja I. and Kamil, Alan C., "Diet Choices of Blue Jays (Cyanocitta cristata) as a Function of Time Spent Foraging" (1988). Papers in Behavior and Biological Sciences. 75.

https://digitalcommons.unl.edu/bioscibehavior/75

This Article is brought to you for free and open access by the Papers in the Biological Sciences at DigitalCommons@University of Nebraska - Lincoln. It has been accepted for inclusion in Papers in Behavior and Biological Sciences by an authorized administrator of DigitalCommons@University of Nebraska - Lincoln. 
Published in Journal of Comparative Psychology 102:3 (1988), pp. 230-235; doi: 10.1037/0735-7036.102.3.230

Copyright ( 1988 American Psychological Association. Used by permission. "This article may not exactly replicate the final version published in the APA journal. It is not the copy of record." http://www.apa.org/journals/jcp/

This research was supported by National Science Foundation Grant BNS-8418721. We thank Betsy Frederick for her invaluable assistance in conducting the experiment, and Kevin Clements, Deborah Olson, and anonymous reviewers for comments on the manuscript.

Submitted July 16, 1987; revised April 22, 1988; accepted April 22, 1988.

\title{
Diet Choices of Blue Jays (Cyanocitta cristata) as a Function of Time Spent Foraging
}

\author{
Sonja I. Yoerg and Alan C. Kamil
}

University of Massachusetts, Amherst

Corresponding author - Sonja I. Yoerg, Department of Psychology, University of Massachusetts, Amherst, Massachusetts 01003

\begin{abstract}
Optimal diet theory predicts choices among prey types. With sequential prey encounters, less profitable prey types may be rejected immediately because rejecting the prey item at hand increases the probability of encountering more profitable types. However, Lucas (1985) argued that at the end of a foraging bout, all encountered prey types should be accepted because the opportunity to encounter more profitable types is limited. We tested Lucas's prediction in a simulation, allowing blue jays to hunt for two moth types differing in profitability. During the last min of both 10- and 20-min foraging bouts, the less profitable type was attacked more often than during the middle of the bouts; this is an end-of-the-bout effect. The less profitable type was also attacked more often at the beginning of the bouts; this is probably a sampling effect. Jays appear to integrate information about time spent foraging with information about relative prey profitability.
\end{abstract}

Optimal diet theory (e.g., Charnov, 1976; MacArthur \& Pianka, 1966; Schoener, 1971) predicts when animals should favor one prey type over others. Choices are partially a function of prey profitability. Profitability is usually assessed as expected energy intake per unit of handling time (the time spent capturing, subduing, and preparing the item for ingestion) and is a dimension on which prey types may be ranked. Theory and data have shown that the inclusion of a prey type in the diet depends not on that prey type's abundance but on the probability of encountering prey items of a higher rank (e.g., Goss-Custard, 1981; Werner \& Mittelbach, 1981). If encounters with prey items are sequential, the animal is choosing whether to attack that item or search further; we will refer to measures of this choice as the attack probability. Searching further usually results in opportunities to attack other types of prey. Indeed, if the disparity in profitability among prey types is large enough, lower ranked types may be rejected (not attacked) as soon as they are recognized. That is, attack probabilities on lower ranked types decrease as their value relative to higher ranked types decreases. Rejection of less profitable types is predicted because opportunities to encounter higher ranking prey types are thereby increased.

Lucas (1985) extended the predictions of the basic diet choice model by providing a theoretical analysis of the effects of different sorts of time constraints on diet choice. The aim of our study was to test one prediction of his analysis - the end-of-the-bout effect. A bout is defined as an uninterrupted interval of foraging. According to Lucas, when the length of the foraging bout is relatively predictable, either because it is stable or because its termination is signalled, an efficient forager should be less selective among different prey types at the very end of the bout. That is, the attack probabilities on prey types of different probability should become more similar; in particular, attack probabilities on the less profitable types should increase. The logic is that at the end of the bout there are no more opportunities to encounter more profitable prey types; the choice between attacking and searching is straightforward when there is insufficient time to search. Consequently, the animal should attack whatever prey type is encountered, regardless of its rank.

In our test of the end-of-the-bout effect, we allowed blue jays to hunt for moths in projected images of two types, some depicting moths on oak trees and others depicting moths on birch trees. The relative profitability of the prey types was manipulated by changes in handling time. Attack probabilities on both prey types were monitored throughout bouts. An end-of-the-bout effect would be manifested as an increase in the attack probability on the less profitable type at the very end of the bout.

\section{Method}

\section{Subjects}

Four adult blue jays between 2- and 14-years old and with varied experimental histories served as subjects. They had been taken from their nests at 10-12 days of age and hand-reared in the laboratory. The jays were maintained at $80 \%$ of their free-feeding weight throughout the experiment by feedings of turkey starter and mynah bird pellets each afternoon.

\section{Apparatus}

The apparatus was a modified operant chamber, $34.0 \mathrm{~cm}$ wide $\times$ $30.0 \mathrm{~cm}$ high $\times 35.5 \mathrm{~cm}$ deep. A food cup was located in the center of one wall, $1.3 \mathrm{~cm}$ above the floor. Reinforcers, half pieces of Tenebrio larvae, were delivered to the food cup by a Davis UF-100 
universal feeder mounted on top of the chamber. Whenever food was delivered, the food cup was illuminated by a small $24-\mathrm{V}$ bulb. A 24-V white house light, located above the cup, was lit continuously during all sessions.

A rectangular pecking key $(11.4 \times 7.5 \mathrm{~cm})$ was mounted with its center $9.9 \mathrm{~cm}$ to the left of the cup, $16.2 \mathrm{~cm}$ above the floor. Behind this key was a programmable Kodak stimulus projector. Three small round pecking keys (each $2.5 \mathrm{~cm}$ in diameter) were located $4.7 \mathrm{~cm}$ below the bottom edge of the rectangular key. Only the middle of these was used in this study; its center was $9.9 \mathrm{~cm}$ to the left of the food cup. A small stimulus projector was mounted behind this key. Masking noise was produced by a ventilating fan and white noise played through a small speaker behind the stimulus panel. A 1.2-cm-diameter perch was located parallel to and $10 \mathrm{~cm}$ in front of the panel, $4 \mathrm{~cm}$ above the floor. A jay on this perch was approximately at eye level with the center of the rectangular key.

Two types of slides were projected onto the rectangular key. Birch slides contained two birch logs photographed against a background of grey cement. Oak slides contained two oak logs photographed against a background of particle board. One third of the birch slides also included a Catocala relicta moth pinned to one of the logs; birch is the typical resting substrate of this species. One third of the oak slides included a C. retecta moth; oak is this species' preferred substrate. Within each slide type (oak or birch), a moth appeared equally often in each vertical third of each log. Slides with moths will be referred to as positives. Those without moths will be referred to as negatives. Both birch and oak positive slides were moderately cryptic. All slides were photographed at a subject-to-camera distance of $1.4 \mathrm{~m}$.

For initial training a set of easier, noncryptic slides was used. The negative slides for this set were produced by photographing two white styrofoam "logs" against a dark background. Positive slides included a C. relicta moth pinned onto one of the logs.

Except during training half of the slides in the projector tray were oak slides and half were birch slides; the probability of encountering the two types was equal. The probability of encountering a moth was the same for the two types, with one third of each type containing a moth. The slides were arranged in random order for each bout with the following restrictions: (a) no more than 10 consecutive slides of the same type, (b) no more than 3 consecutive positive slides of one type, and (c) no more than 5 consecutive negative slides of one type. Ten slide sequences were generated. A new sequence was used every other day, in random order. On the days that sequences were not changed, the bout began in the middle of the slide tray instead of at the beginning.

\section{General Procedure}

At the start of each foraging bout (session), the jay was placed on the perch. The first trial began when the small round key turned yellow. A peck at this key changed the color to green and caused a slide to be projected onto the rectangular key. The jay could make one of two responses at this stage in the trial, (a) peck the round key or (b) peck the rectangular key. If the round key was pecked, the trial ended. Both the round and rectangular keys became dark. A 1-s interval followed during which the slide projector advanced. The next trial began with the illumination of the round key. Each trial was analogous to an entry into a patch that may contain a prey item.

If the jay pecked the rectangular key, the round key became dark and remained inoperative for the remainder of the trial. The latency to peck the rectangular key is the search time. The handling-time interval (see below) began after this peck, whether or not a moth was present in the slide. During this interval pecks to the rectangular key had no effect, though the jays usually did peck at the image. The first peck after the end of the handling-time in- terval ended the trial, and the rectangular key became dark and inoperative. If the slide had been a positive (contained a moth), the feeder operated, and a piece of mealworm was delivered into the food cup. A 1-s interval followed during which the slide projector advanced. If the slide had been a negative (no moth present), no mealworm was delivered and the slide projector advanced immediately. The next trial then began. The correct response for positive slides was to peck the rectangular key. The correct response for negative slides was to peck the round key.

Throughout the experiment the jays were allowed one foraging bout per day, 6 days per week. Foraging bout length information is given below.

\section{Training}

All training bouts lasted for 60 trials. The handling-time interval was 20 s. For 2 of the jays (Jay 74 and Jay 49), initial training with the procedure was conducted with a set of non-cryptic slides (see above). The criterion for entering the next training phase was $90 \%$ correct on both positive and negative slides for 5 consecutive days. Jay 74 reached this criterion in 2 months; Jay 49 reached criterion in 1 month.

The birds were then transferred to cryptic slides. Jay 51 was added to the roster and did not require training with noncryptic slides because of its previous experience. The 3 jays were first trained with cryptic birch slides. The same performance criterion was applied as with the noncryptic slide set. After the birds met criterion (Jay 74, 1 month; Jay 49, 2 months; Jay 51, 2 months), they were trained with only cryptic oak slides. All 3 jays required 2 months of training with oak slides to meet the criterion.

Jay 36 did not require training because it had had experience with both cryptic birch and oak slides in a previous experiment.

\section{Baseline}

As will be explained in detail below, the handling time of one prey type remained stable throughout the experiment, whereas the handling time of the other prey type could vary between foraging bouts. Our goal was to select handling times that resulted in attack probabilities near $50 \%$ for the less profitable type. Achieving this goal would maximize our ability to detect changes in attack probabilities as a function of time in a bout. We could not compute a priori what difference in handling time between the two types would result in an attack probability of $50 \%$ for the less profitable type. This computation depends on the precise travel times (time between trials), search times, and actual handling times (as opposed to the programmed handling times) produced by the birds. For example, with long handling times, the birds slow down, taking longer between trials. This, according to diet theory, should affect the relation between attack probability and the difference in profitability between the prey types. It was imperative that the handling time for the type with the constant profitability be selected carefully.

We conducted an 18-bout baseline period to collect data on travel times, search times, and actual handling times at three different programmed handling times (18, 30, and $42 \mathrm{~s})$. Each bout was randomly assigned one of these handling times that applied to both prey types. Birch and oak slides were mixed in the slide tray according to the sequences described above.

The baseline data were then used to determine the handling time of the prey type with the constant profitability. For all the birds a handling time of $18 \mathrm{~s}$ for the constant prey type was adequate in that the predicted handling time for the less profitable type at an attack probability of $50 \%$ was neither too long (the jays stopped responding with handling times over $1 \mathrm{~min}$ ) nor too close to $18 \mathrm{~s}$ to obscure the difference in profitability. 


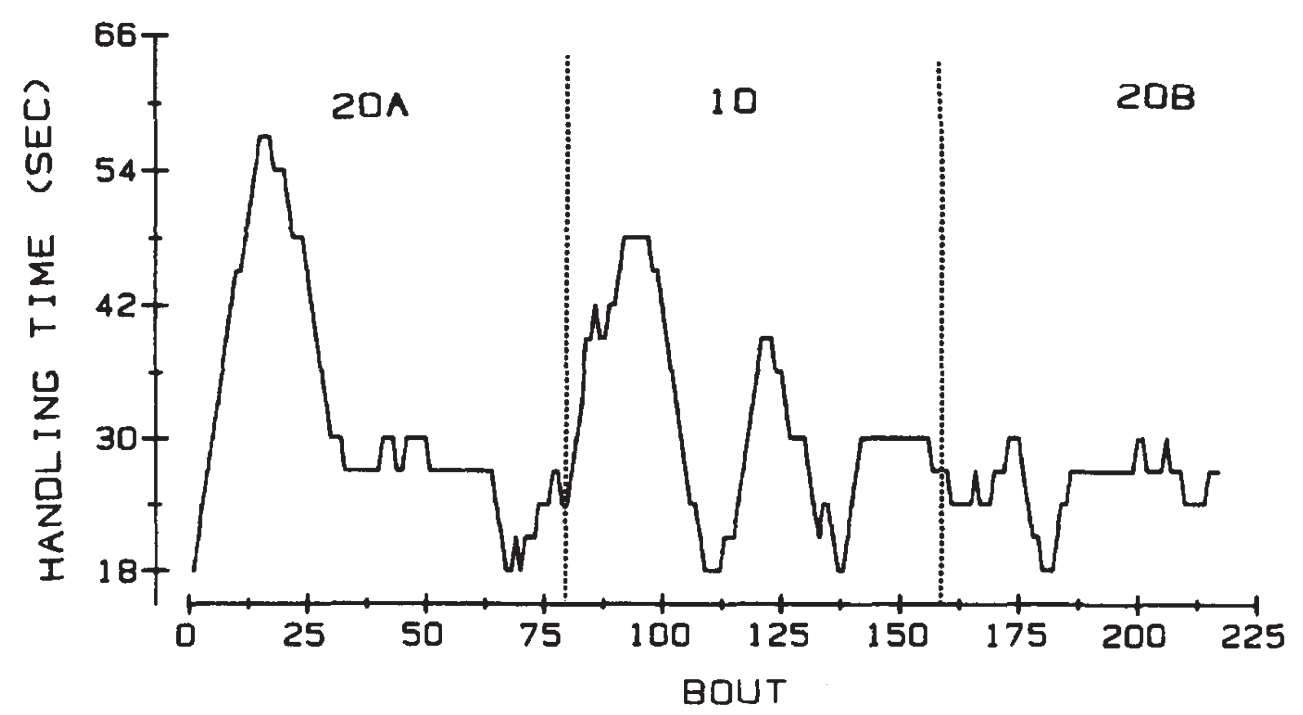

Figure 1. Handling-time values for the less profitable prey type (birch) in each bout of the experiment for Jay 36. (20A, 10, and 20B represent the three conditions.)

\section{Condition 20A}

After baseline the jays were randomly assigned to one of two groups. For Jays 36 and 49 the profitability of the birch slide type was constant; for Jays 51 and 74 the profitability of the oak slide type was constant. Bout length was set at $20 \mathrm{~min}$. Any trial that began before $20 \mathrm{~min}$ had elapsed was allowed to be completed.

The handling time for the constant type for both groups was $18 \mathrm{~s}$. The handling time for the other prey type was also initially set at $18 \mathrm{~s}$ but could vary from bout to bout thereafter. If the attack probability for this prey type was below $34 \%$, the handling time for the subsequent bout was decreased $3 \mathrm{~s}$ unless the handling time was already $18 \mathrm{~s}$. If the attack probability for this prey type was above $66 \%$, the handling time for the subsequent bout was increased $3 \mathrm{~s}$. If the attack probability fell between these values, the handling time remained unchanged. This titration procedure maintained attack probabilities near $50 \%$.

Condition 20A ended when handling time values did not change during 9 out of 10 consecutive bouts, that is, when attack probabilities for the less profitable prey type remained between $34 \%$ and $66 \%$. The number of bouts required to meet this criterion was 72 bouts for Jay 74, 79 bouts for Jay 36, 84 bouts for Jay 49, and 108 bouts for Jay 51 .

\section{Condition 10}

Condition 10 was identical to the previous condition except that bouts terminated after the completion of the last trial that started before $10 \mathrm{~min}$ had elapsed. All jays completed 80 bouts in this condition.

\section{Condition 20B}

Condition 20B was identical to Condition 20A. The criterion for terminating this condition was 40 bouts in which the attack probability for the less profitable type was between $34 \%$ and $66 \%$. The number of bouts required to meet this criterion was 55 bouts for Jay 51 , 58 bouts for Jay 36, 66 bouts for Jay 74, and 78 bouts for Jay 49 .

\section{Results}

The attack probability on the more profitable prey type was high throughout the experiment, typically $100 \%$. In contrast, the attack probability on the less profitable type was almost always below $100 \%$ and varied considerably within bouts. The jays correctly rejected no-prey slides of both types on nearly every encounter. Our analyses focus on the within-bouts variation in the probability of attacking the lower-ranking prey type.

\section{End-of-the-Bout Effect}

If the jays were anticipating the end of the bout, the attack probabilities for the less profitable prey type should have increased just as the bout was terminating. We calculated the attack probability for the last min in each bout for each bird. This was done by starting at the end of the bout and including all trials that ended within $60 \mathrm{~s}$ of the end of the bout. This probability was then divided by the mean attack probability for the less profitable type during the middle $60 \%$ of the bout, the middle $12 \mathrm{~min}$ for $20-\mathrm{min}$ bouts and the middle $6 \mathrm{~min}$ for 10 -min bouts. The resulting ratio, the attack ratio, is a statistic expressing how much more (if greater than 1.0) or less (if less than 1.0) likely the jays were to attack the less profitable prey type at the end of the bout relative to the middle of the bout.

Because of the titration procedure the attack probabilities during the middle of the bout were usually close to $50 \%$. However, during a significant proportion of bouts, the mean attack probability across the entire bout was not within the $34-66 \%$ range (i.e., the handling time would change the next day). Figure 1 and 2 show handling times for the less profitable type for two of the birds in every bout. These fluctuations in handling times correspond to fluctuations in the at- 


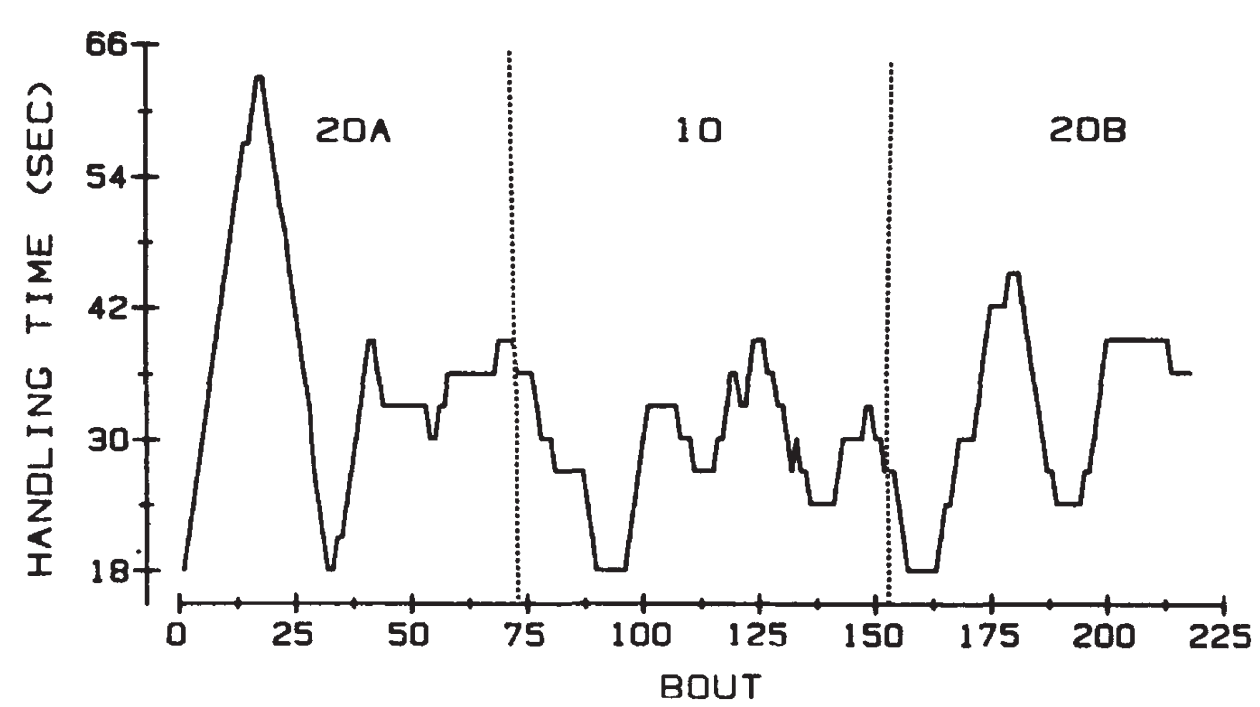

Figure 2. Handling-time values for the less profitable prey type (oak) in each bout of the experiment for Jay 74. (20A, 10, and 20B represent the three conditions.)

tack probability on the less profitable type as a result of the titration procedure. It was possible that our ability to detect the end-of-the-bout effect would be affected by the overall attack probability for that bout. Consequently, in our initial analyses of attack ratios, we compared the size of the effect (a) when all bouts were included in the analysis and (b) when only those bouts with mean attack probabilities of 34\%-66\% were included. The size of the effect did not differ. This makes intuitive sense: The attack ratio, because it is a within-bouts measure, should be relatively robust with respect to daily variation in mean attack probability. In all subsequent analyses all bouts were included.

The mean attack ratios for each bird during the last 30 bouts in each condition are given in Table 1. (In all subsequent analyses, unless otherwise specified, the last 30 bouts in each condition were used.) The mean attack ratios for each condition were all significantly greater than 1.0: None of the $95 \%$ confidence intervals around the mean included 1.0. The jays were more likely to attack the less profitable prey type during the last min of the bout than during the middle of the bout. Mean attack ratios did not differ between conditions; Condition 10 versus 20A, $F(1,3)=7.61$, $n s$; Condition 10 versus $20 \mathrm{~B}, F(1,3)=1.28$, ns; Condition $20 \mathrm{~A}$ versus $20 \mathrm{~B}, \mathrm{~F}(1,3)=3.51$, ns.

The deviation of the attack ratios from 1.0 is one way to evaluate the size of the end-of-the-bout effect. Perhaps a better way, especially for Condition 10, is to compare for each bird the attack ratio calculated as described above with the attack ratio calculated from the first $10 \mathrm{~min}$ of the $20-\mathrm{min}$ bouts. In other words, we treated the first $10 \mathrm{~min}$ of 20-min bouts exactly as we treated 10-min bouts, dividing the attack probability in the 10th min by the mean attack probability during the middle $6 \mathrm{~min}$ of the first half of the bout (Min 3-8). A comparison between the attack ratios from the first half of 20-min bouts and the attack ratios from 10-min bouts controls for the possible effects of satiation.

The attack ratios for the 10-min bouts were significantly higher than the attack ratios for the first half of the 20-min bouts of Condition 20A, F $(1,3)=19.80, p<.05$ (see Table 1 ). The attack ratios for the first half of the bouts of Condition 20B did not differ from those for the 10-min bouts, $F$ $(1,3)=1.28$, ns. The mean differences between the two ratios were 0.31 comparing the first $10 \mathrm{~min}$ of Condition 20A and 0.14 comparing the first $10 \mathrm{~min}$ of Condition $20 \mathrm{~B}$.

The results are similar if the attack ratios from each of the 20-min conditions are compared to the attack ratios from the first half of that condition. For Condition 20A the mean difference between the ratios was 0.85 . The jays were significantly more likely to attack the less profitable prey during the last min of the bout than during the 10th min, $F(1,3)=43.61, p<.01$. For Condition 20B the mean difference between the ratios was 0.28 . In this condition the jays were not more likely to attack the less profitable prey during the last min than during the 10th $\min , F(1,3)<1$, ns.

\section{Beginning-of-the-Bout Effect}

The jays were also more likely to attack the less profitable prey type at the beginning of the bout. Table 2 shows the mean attack ratios for the last 30 bouts in each condition, in which the attack probability during the 1st min was divided by the attack probability during the middle of the bout. The ratios were significantly greater than 1.0 for each condition: 95\% confidence intervals around the mean do not include 1.0.

Table 1. Mean End-of-Bout Attack Ratios for Each Condition and for the First Half of 20-Min Conditions

\begin{tabular}{lccc}
\hline Condition & $\begin{array}{c}\text { Mean attack } \\
\text { ratio }\end{array}$ & SD & $\begin{array}{c}95 \% \text { confidence } \\
\text { interval }\end{array}$ \\
\hline $20 \mathrm{~A}$ & 1.81 & 0.36 & $1.46-2.17$ \\
10 & 1.28 & 0.25 & $1.03-1.53$ \\
$20 \mathrm{~B}$ & 1.42 & 0.19 & $1.24-1.60$ \\
First half 20A & 0.97 & 0.25 & $0.72-1.21$ \\
First half 20B & 1.14 & 0.18 & $0.97-1.32$ \\
\hline
\end{tabular}

Values are from the last 30 days of each condition. 
Table 2. Mean Beginning-of-Bout Attack Ratios for Each Condition

\begin{tabular}{lccc}
\hline Condition & $\begin{array}{c}\text { Mean attack } \\
\text { ratio }\end{array}$ & $S D$ & $\begin{array}{c}95 \% \text { confidence } \\
\text { interval }\end{array}$ \\
\hline $20 \mathrm{~A}$ & 2.63 & 0.87 & $1.78-3.48$ \\
10 & 1.72 & 0.54 & $1.19-2.25$ \\
$20 \mathrm{~B}$ & 1.85 & 0.46 & $1.40-2.30$ \\
\hline
\end{tabular}

Values are from the last 30 days of each condition.

We thought of two explanations for this effect. First, the birds were hungrier at the beginning of the bout and might therefore attack whatever prey type is encountered. Once several mealworms had been consumed, their choices became more discriminating. Alternatively, the high probability of attack on the less profitable prey type might represent sampling (e.g., Krebs, Kacelnik, \& Taylor, 1978; Lima, 1984; Shettleworth, Krebs, Stephens, \& Gibbon, in press). Presumably the function of sampling in this situation was to obtain information on handling-time values, which could change from bout to bout.

The two hypotheses make different predictions about the size of the effect across conditions. If attack probabilities were high because of hunger, there should be no difference between conditions in the magnitude of the effect. In other words, the jays were just as hungry at the start of 10min bouts as they were at the start of 20 -min bouts. If, however, high initial attack probabilities indicated sampling, more sampling would be expected in 20-min bouts. The information gained from sampling would be more valuable in a longer bout: Given that a sampling period must be long enough to obtain a good estimate of the parameter evaluated, that period is a smaller proportion of the bout in longer bouts. The cost of a sampling period is fixed, but in a longer bout there are more opportunities to use the information derived from sampling (Stephens \& Krebs, 1987).

The attack ratios shown in Table 2 do not differ between conditions: Condition $20 \mathrm{~A}$ versus $20 \mathrm{~B}, F(1,3)=2.40$, ns; Condition 20A versus 10, F $(1,3)=2.33$, ns; Condition 20B versus $10, F(1,3)<1$, ns. However, it is not unreasonable to suppose that a sampling period of $1 \mathrm{~min}$ may be too short to yield information of value, especially because on the average less than 1 positive slide of each type was encountered. We chose to analyse the first 3 min of each bout. The attack probabilities during the first $3 \mathrm{~min}$ of Condition 20A $(M=$ $0.699 \pm 0.127)$ did not differ from the attack probabilities during the first $3 \mathrm{~min}$ of Condition 20B $(M=0.726 \pm 0.157), F$ $(1,3)<1$, ns. We therefore combined the data from the two 20-min conditions and compared them to the attack probabilities from the $10-\mathrm{min}$ condition $(M=0.613 \pm 0.155)$. The jays were more likely to attack the less profitable prey type during the first $3 \mathrm{~min}$ of 20-min bouts than during the first 3 min of 10-min bouts, $F(1,3)=10.92, p<.05$.

\section{Rates of Encounter}

The jays could control, within limits, the rate at which they made prey choices by delaying either the initiation of a trial or the decision to attack or reject. Any systematic changes in rates of encounter within bouts would complicate the interpretation of the effects reported.

Rates of encounter (mean number of trials per minute) during the 1st min, the middle 6 (Condition 10) or 12 (Conditions 20A and 20B) min, and the last min were calculated for the last 30 days in each condition. There were no differences in rates of encounter between the two 20-min conditions: first, $F(1,3)<1$, ns; middle, $F(1,3)=9.36$, ns; last, $F$ $(1,3)<1$, ns. An analysis of the combined data from the $20-$ min conditions revealed no within-bouts differences in encounter rate, $F(2,6)=1.26$, ns. The jays completed a mean of 6.2 trials during the 1 st min, 7.0 trials per minute during the middle $12 \mathrm{~min}$, and 5.7 trials during the last min of the 20 -min bouts. A similar analysis of the encounter rates during the 10-min condition yielded a similar null result, $F(2$, $6)<1$, ns. In this condition the jays completed a mean of 6.0 trials during the 1 st $\mathrm{min}, 6.8$ trials per minute during the middle $6 \mathrm{~min}$, and 6.6 trials during the last $\mathrm{min}$.

\section{Discussion}

As diet selection models predicted (e.g., Charnov, 1976; MacArthur \& Pianka, 1966; Schoener, 1971), the probability of the jays' attacking the less profitable prey type depended on its relative profitability, that is, on its handlingtime value. Given the choice between attacking the prey item encountered and rejecting that item and searching further, the jays were more likely to reject the prey as its net energetic value decreased. This basic finding is not novel (e.g., reviews by Krebs, Stephens, \& Sutherland, 1983; Pyke, Pulliam, \& Charnov, 1977).

As Lucas (1985) predicted, the jays were more likely to attack the less profitable prey type at the very end of the foraging bout as compared to the middle of the bout; they demonstrated an end-of-the-bout effect. This presumably occurred because given the fixed bout length within conditions, the jays anticipated the end of the bout. As the bout was about to end, they lost opportunities to encounter the higher ranking (more profitable) prey type. This resulted in an increase in the relative value of the less profitable prey type.

Only one of our analyses failed to provide strong support for the presence of an end-of-the-bout effect: The analysis that compared attack ratios during the first half of 20min bouts with attack ratios during 10-min bouts produced equivocal results. Consistent with our other analyses, the ratios from the first half of the bouts in Condition 20A were lower than those from Condition 10. That is, the 10th min of those 20-min bouts was not similar to the last min of the 10-min bouts. However, we failed to obtain the same result when the first half of the bouts in Condition 20B was used. The source of this discrepancy is unclear.

We also detected a substantial increase in attack probability on the lower ranked prey type at the beginning of bouts. Because attack probabilities during the first 3 min were higher during 20 -min than during 10 -min bouts, it is unlikely that hunger is an adequate explanation. It seems possible that the birds used the initial portion of longer 
bouts to gather information. Because handling-time values were the only source of change between bouts, we suspect that this was the information they sought, though we have no direct evidence bearing on this. The information gleaned from a sampling period is more useful in longer bouts because the sampling interval represents a smaller proportion of the total foraging time; the potential benefit derived from the information obtained is greater in longer bouts because there is more time to use it. The cost of obtaining the information is the same regardless of bout length. Hence, economic considerations lead to the prediction that our experiment bore out.

The end-of-the-bout effect implies that the jays integrated information about foraging time with information about the relative value of prey types. The sampling effect implies that the jays assessed the potential value of the information that could be obtained by accepting lower ranking prey types at the start of the bout. Together, these results indicate that blue jays used the time spent foraging (or the time remaining for foraging) to guide diet choice in a sophisticated manner.

\section{References}

Charnov, E. L. (1976). Optimal foraging: Attack strategy of a mantid. American Naturalist, 110, 141-151.

Goss-Custard, J. D. (1981). Feeding behavior of redshank, Tringa tetanus (L.), in relation to prey density. Journal of Animal Ecology, 46, 1-19.
Krebs, J. R., Kacelnik, A., \& Taylor, P. (1978). Test of optimal sampling by foraging great tits. Nature, 275, 27-31.

Krebs, J. R., Stephens, D. W., \& Sutherland, W. J. (1983). Perspectives in optimal foraging. In A. H.Brush \& G. A.Clark (Eds.), Perspectives in ornithology (pp. 165-215). Cambridge, England: Cambridge University Press.

Lima, S. (1984). Downy woodpecker foraging behavior: Efficient sampling in simple stochastic environments. Ecology, 65, 166-174.

Lucas, J. R. (1985). Time constraints and diet choice: Different predictions from different constraints. American Naturalist, 126, 680-705.

MacArthur, R. H., \& Pianka, E. R. (1966). On optimal use of a patchy environment. American Naturalist, 100, 603-609.

Pyke, G. H., Pulliam, H. R., \& Charnov, E. L. (1977). Optimal foraging theory: A selective review of theory and tests. Quarterly Review of Biology, 52, 137-154.

Schoener, T. W. (1971). Theory of feeding strategies. Annual Review of Ecology and Systematics, 2, 239-404.

Shettleworth, S. J., Krebs, J. R., Stephens, D. W., \& Gibbon, J. (in press). Tracking a fluctuating environment: A study of sampling. Animal Behaviour.

Stephens, D. W., \& Krebs, J. R. (1987). Foraging theory. Princeton, NJ: Princeton University Press.

Werner, E. E., \& Mittelbach, G. G. (1981). Optimal foraging: Field tests of diet choice and habitat switching. American Zoologist, 21, 813-829. 